\title{
A Study on Fight Against COVID-19 from Latest Technological Intervention
}

\author{
Enoch Arulprakash $^{1}$ (D) $\cdot$ Martin Aruldoss ${ }^{1}$
}

Received: 6 August 2020 / Accepted: 10 August 2020 / Published online: 19 August 2020

(c) Springer Nature Singapore Pte Ltd 2020

\begin{abstract}
Uncontrolled spread of pandemic COVID-19 in India and across the globe over several months, created an impact as never before any pandemic would have created. This certainly demands a technological intervention from all possibility to overcome the situation and lead a normal life as early as possible. AI/Machine learning responds to the situation, through inspecting different aspects of the pandemic. This paper analyses and studies those aspects, (I) Quarantine and statistical aspect: Quarantine potentially affected candidates (person who is in touch, travel history) through Data analytics/Machine learning. (II) Diagnosis and Treatment aspect: Early detection and fast treatment will save lives. Diagnosis using deep learning assists radiologist from saving their effort and time to a greater extent and arrives faster conclusion. (III) Prevention aspect: Monitoring and enforce social distancing through visual social distancing using deep learning and Computer vision.
\end{abstract}

Keywords Technological intervention $\cdot$ Artificial intelligence $\cdot$ Data analytics $\cdot$ Machine learning $\cdot$ Object detection

\section{Introduction}

Spread of COVID-19 in India and across the globe over several months insists the countries to call for a complete lockdown which was affecting the normal life of humans from all the spectrum of the life, including daily labours to the multinational business, thereby affecting the world economy, and deprived as never before, since the 1930s [13]. But, the lockdowns failed to eradicate pandemic instead, raised another set of problems like ceaselessness, unemployment, poverty, etc., and leaving a fear about the future. However, fight for the COVID-19 is unstoppable, to safeguard human life until the vaccine is invented, that is being the prime most challenge for the Pharmaceutical sector. AI/Machine learning responds to the situation, through inspecting different

This article is part of the topical collection "Advances in Computational Approaches for Artificial Intelligence, Image Processing, IoT and Cloud Applications" guest edited by Bhanu Prakash K N and M. Shivakumar.

Enoch Arulprakash

enocharulprakash03@gmail.com

Martin Aruldoss cudmartin@gmail.com

1 Department of Computer Science, CUTN, Thiruvarur, India aspects of the pandemic. This paper analyses and studies those aspects in detail like, (I) Quarantine and Statistical Aspect Quarantine potentially affected candidates (person who is in touch, travel history) through Data analytics/ Machine learning [2, 18]. (II) Diagnosis and Treatment Aspect Early Diagnosis and fast treatment will save lives. Diagnosis using deep learning assists radiologist from saving their effort and time to a greater extent and arrives faster conclusion [8, 20]. (III) Prevention Aspect Machine learning and Computer vision as an aid for drug discovery and Monitoring and enforce social distancing through visual social distancing $[4,15]$.

Paper is organised as section "Quarantine and Statistical", section "Diagnosis and Treatment", section "Prevention", section "Conclusion".

\section{Quarantine and Statistical Aspect}

COVID-19 shows very mild symptoms initially like cold or cough and gradually leading to severe diseases such as Middle East Respiratory Syndrome and Acute Respiratory Syndrome, causing difficult to detect the virus in the early stage. It can be easily out-broken from the affected person when he/she coughs, speaks or sneezes from a circumference of one metre. The virus affects anyone without any 
constraint, causing a major risk to the person already suffering from high blood pressure, heart and lung problems, diabetes, or cancer [5].

Direct contacts of the infected have to be quarantined and monitored for 14 days as per World Health Organisation (WHO) to prevent further outbreak [5]. To Quarantine potential affected candidates is a challenging task, since infected may have domestic or international travels, which results in analysing a large amount of data to draw possible inference for a potential affected candidates; manually accomplishing it will lead to consuming a lot of time. Data analytics and machine learning provide a powerful method to inference data from multiple perspectives, essential for suspicion and quarantine [9, 18]. Machine learning can also assist in forecasting from historical data; the machine learning model predicts the future concerning time and geographical area. Thereby, authorities take various precautionary measures to prevent the outbreak and prepare various disciplines for action against COVID-19 in their regime. For example, Machine learning model BlueDot accurately predicted the threat of COVID-19 even before WHO declared publicly [1]. Similarly, other ML models were also effective in forecasting like Healthmap [12], Google Flu Trends [7], etc. Furthermore, ML Models are also used for tracking the COVID-19 cases across the globe in Data Dashboards like UpCode, NextStrain and Healthmap [2], effective in drawing useful insight like the number of recoveries, number of new cases and number of deaths, which part of the region controlled the outbreak, etc.

\section{Diagnosis and Treatment}

\section{Diagnosis}

Early diagnosis and fast treatment will save a life; there are various methods to diagnose COVID-19, like Nucleic Acid Test (NAT) and CT scans. Among these, CT scan is most effective in terms of early results, cost and accessing the degree of severity of the infected. Determining the degree of severity is essential, since not all diagnosed patient requires intensive care, it helps for planning effective utilisation of medical resources [5].

The outbreak is increasing day by day and it is causing overload and burden to the radiologist. However, diagnosing using deep learning as a potential to compete forefront radiologist [14, 19]. Thereby, saves radiologists effort and time to a greater extent and arrives faster conclusion. Besides that, deep learning diagnosis methods results are faster and crucial for quick treatment. There are several publically available deep learning models trained from various patients' data, models like COVID-Net, CAD4COVID [11,
19], etc., which can be utilised directly or retrained with the new patients for enhanced prediction.

Also, diagnosis is applicable in the Smartphone; Smartphone can easily read CT scan images, and analyse using its various sensors (temperature sensor, inertial sensors, humidity sensor, and wireless chipsets/sensors). Today, everybody has a Smartphone, providing provision to examine, even in a remote village. Moreover, it can be used for initial screening [10].

\section{Treatment}

Discovering a drug is a major challenge to the Pharmaceutical sector; a study suggests that complete vaccine for a virus would require at least 18-months [12]. Thus, Alternative drugs are the only way for the treatment; again selecting an optimal alternative drug carries considerable risk due to scientific investigation, trails and regulations. However, ML models provide a mechanism to analyse data and provide useful insight and prediction which is used for suggesting an appropriate alternative for treatment. ML models suggested alternative vaccines like atazanavir [3] and baricitinib [9, 12]; further, these drugs are used for treating the COVID19-affected patients.

\section{Prevention Aspect}

Prevention aspect can be viewed from two perspectives.

\section{Complete Cure of COVID-19}

Discovering a vaccine for a complete cure is a challenging and time-consuming task. Notwithstanding, AI has the potential to accelerate the process significantly, discovering the protein structure plays a crucial role in the discovery of a vaccine. Google's Deep-mind $[6,16]$ has declared COVID-19's protein structure. However, Google is not certain about the structure. Notwithstanding, prediction can be instrumental in drug discovery.

\section{Visual Social Distancing}

Since pandemic outbreak is severe and uncontrollable, now the world started to cope with the pandemic with safety measures. Enforcing the regulation and strictly monitoring the violation are necessary. But, continuous human monitoring will lead to cognitive load and prone to error, furthermore inefficient in finding violations in crowded regions. Strict social distancing and precautionary measures like wearing a mask are the primary concern to carry out dayto-day activities during the outbreak. To confront the situation and provide strict monitoring, computer vision-object 
detection is an optimal solution. Experimentary results highlights efficient performance of deep learning based framework, for monitoring the social distancing via object detection and tracking approaches, with balanced FPS and mAP score $[4,17]$. However, computer vision-object detection is in the preliminary stage of the development to widely enforce visual social distance monitoring. But these methods have already proven high accuracy in digital object detection and tracking. Moreover, there are certain parts of the world, where visual social distancing has been enforced like in south china at airports and train stations across the regions; special cameras with facial recognition features are used to scan the crowds for high temperature. This visual camera identifies the person who has got a high temperature along with whether a person is wearing a mask or not [12].

\section{Conclusion}

Undoubtedly, technological intervention for the fight against Covid-19 is crucial and significant. But, with the advance technological enhancement and its intervention left the contest to continue with a hope to uproot in the future. In certain aspects, convention models are preferred than Artificial Inteligence (AI) models like Susceptibles infectives removed (SIR) models as the lack of data, unwanted data and inexperience make AI less reliable.

However, AI has the potential to fight from all aspects, assist people from various disciplines in creating awareness, carry out safety measures, effective management of resources and help authorities to enforce strict regulations. AI alone has the potential to assist all the sectors to fight effectively, against any natural calamities as they reveal from the scientific perspective.

\section{References}

1. BlueDot: Outbreak Risk Software.

2. The best, and the worst, of the coronavirus dashboardsIMIT Technology Review.

3. Beck BR, Shin B, Choi Y, Park S, Kang K. Predicting commercially available antiviral drugs that may act on the novel coronavirus (SARS-CoV-2) through a drug-target interaction deep learning model. Computat Struct Biotechnol J. 2020;18:784-90.

4. Cheung M, She J. Detecting social signals in user-shared images for connection discovery using deep learning. IEEE Trans Multimed. 2020;22(2):407-20.

5. Culp WC. Coronavirus disease 2019. A \& A Practice. 2020;14(6):e01218.

6. DeepMind. Computational predictions of protein structures associated with COVID-19. DeepMind; 2020. https://deepmind.com/ research/open-source/computational-predictions-of-protein-struc tures-associated-with-COVID-19. Accessed 14 Aug 2020.

7. Kandula S, Shaman J. Reappraising the utility of Google flu trends. PLoS Comput Biol. 2019;15(8):1-16.

8. Kiyokawa T, Tomochika K, Takamatsu J, Ogasawara T. Fully automated annotation with noise-masked visual markers for deep-learning-based object detection. IEEE Robot Autom Lett. 2019;4(2):1972-7.

9. Kumar A, Gupta PK, Srivastava A. A review of modern technologies for tackling COVID-19 pandemic. Diabetes Metab Syndr: Clin Res Rev. 2020;14(4):569-73.

10. Maghdid HS, Ghafoor KZ, Sadiq AS, Curran K, Rabie K. A novel AI-enabled framework to diagnose coronavirus COVID 19 using smartphone embedded sensors: design study. 2020. arXiv preprint arXiv:2003.07434.

11. Murphy K, Smits H, Knoops AJG, Korst MBJM, Samson T, Scholten ET, Schalekamp S, Schaefer-Prokop CM, Philipsen RHHM, Meijers A, Melendez J, van Ginneken B, Rutten M. COVID-19 on the chest radiograph: a multi-reader evaluation of an AI system. Radiology. 2020;296:E166-72.

12. Naudé W. Artificial intelligence vs COVID-19: limitations, constraints, and pitfalls. AI Soc. 2020. https://doi.org/10.1007/s0014 6-020-00978-0.

13. Chigoziri PU. Appraisal of coronavirus on global economy: "Ineffectiveness of economic growth and development in global south". NOLEGEIN-J Bus Risk Manag. 2020:1-18

14. Ozturk T, Talo M, Yildirim EA, Baloglu UB, Yildirim O, Rajendra Acharya U. Automated detection of COVID-19 cases using deep neural networks with X-ray images. Comput Biol Med. 2020;121:103792.

15. Punn NS, Sonbhadra SK, Sonali Agarwal S. Monitoring COVID19 social distancing with person detection and tracking via finetuned YOLO v3 and Deepsort techniques. 2020:1-10. arXiv preprint arXiv:2005.01385.

16. Silver D, Hubert T, Schrittwieser J, Antonoglou I, Lai M, Guez A, Lanctot M, Sifre L, Kumaran D, Graepel T, Lillicrap T, Simonyan K, Hassabis D. A general reinforcement learning algorithm that masters chess, Shogi, and go through self-play. Science. 2018;362(6419):1140-4.

17. Soures N, Chambers D, Carmichael Z, Daram A, Shah DP, Clark K, Potter L, Kudithipudi D. SIRNet: understanding social distancing measures with hybrid neural network model for COVID-19 infectious spread. 2020. arXiv preprint arXiv:2004.10376.

18. Jason Wang C, Ng CY, Brook RH. Response to COVID-19 in Taiwan: big data analytics, new technology, and proactive testing. JAMA. 2020;323(14):1341-2.

19. Wang L, Wong A. COVID-Net: a tailored deep convolutional neural network design for detection of COVID-19 cases from chest X-ray images. 2020:1-12. arXiv preprint arXiv:2003.09871.

20. Xiangjun W, Hui H, Niu M, Li L, Wang L, He B, Yang X, Li L, Li $\mathrm{H}$, Tian J, Zha Y. Deep learning-based multi-view fusion model for screening 2019 novel coronavirus pneumonia: a multicentre study. Eur J Radiol. 2020;128(April):1-9.

Publisher's Note Springer Nature remains neutral with regard to jurisdictional claims in published maps and institutional affiliations. 